\title{
Latitudinal distribution of the equatorial plasma bubbles: Altitude variability
}

\author{
L.N. Sidorova * \\ Pushkov Institute of Terrestrial Magnetism, Ionosphere and Radiowave Propagation (IZMIRAN), Kaluzhskoe \\ shosse, d.4, 108840 Moscow, Troitsk, Russia
}

\begin{abstract}
In this paper, the latitudinal distributions of the equatorial plasma bubbles (EPBs) and their variability with the observation heights were studied. For this purpose, a detailed comparative analysis of the latitudinal histograms of the EPB occurrence probability obtained at the different altitudes was performed. The data from the ISS-b $(\sim 972-1220 \mathrm{~km})$ ROCSAT-1 $(\sim 600 \mathrm{~km})$ and AE-E $(\sim 350-475 \mathrm{~km})$ spacecrafts were used for this purpose. The EPB structures were identified from the ISS-b data as the $\mathrm{He}^{+}$density depletions, and from the ROCSAT-1 and AE-E data as the ion density $\left(\mathrm{N}_{i}\right)$ depletions. Different seasons during the years of the high solar activity conditions were under consideration. It was obtained that as the observation height increases from $\sim 600 \mathrm{~km}$ to $\sim 1000 \mathrm{~km}$, the mid-latitude maxima in addition to the equatorial maximum develop in the histograms of the EPB occurrence probability. This feature was most pronounced in both the hemispheres of the equinox periods and in the winter hemispheres. At the altitudes of the upper topside ionosphere $(\sim 972-1220 \mathrm{~km}$, ISS-b), the values of the mid-latitude maxima of the EPB occurrence probability become comparable or begin to dominate in relation to the equatorial maximum. This feature is typical for all seasons of the observations. Moreover, it was obtained that the latitudinal positions of the mid-latitude maxima of the EPB occurrence probability shift to the equator with increase in the observation heights. The latter is in good accordance with the latitudinal courses of the magnetic field tubes along which the plasma bubbles that have already reached their ceiling heights are stretched.
\end{abstract}

Keywords : Equatorial plasma bubbles; Latitudinal occurrence probability; Topside ionosphere; Fregion ionosphere

\section{Introduction}

Intensive study of the ionosphere plasma irregularities controlled by the electrodynamics processes of the equatorial region started in the seventies of the last century. According to the radar [Tsunoda, 1980; Woodman and La Hoz, 1976], ionosonde [Abdu et al., 1983] and satellite measurements the class of the irregularities with the plasma density depleted by several times to several orders of magnitude was identified. Such depletions can occupy several hundred kilometers in the east-west direction, thousands

*corresponding author:lsid@izmiran.ru 
of kilometers in the north-south direction and can rise to the topside ionosphere heights. The plasma irregularities of this type are called as the equatorial plasma bubbles (EPBs).

Some attempts have been made to obtain the latitudinal occurrence probability of the EPBs at the different altitudes based on the spacecraft and ground-based observations. However, insufficient measurement data did not allow getting the reliable conclusions about the EPB latitudinal distributions and their variability with increasing observation height [Abdu et al., 2000]. That is why the mentioned problem remained poorly investigated for a long time.

For example, the latitudinal histograms of the EPB occurrence probability were obtained at the bottom side of the F-region in the different seasons during the years of the high solar activity (AE-E spacecraft data, $(\sim 300-475 \mathrm{~km})$ [Kil and Heelis, 1998]. These plots were compared with the plots obtained at the altitudes of $\sim 600 \mathrm{~km}$ (ROCSAT-1 spacecraft) [Su et al., 2006]. It was obtained that there is the basic similarity between these plots in the equatorial and low-latitudinal regions. However, there are fundamental differences between them in the mid-latitude region [Su et al., 2006]. So, in addition to the equatorial maximum the additional mid-latitudinal maxima of the EPB occurrence probability appear in the histograms, which are typical for the altitudes of $\sim 600 \mathrm{~km}$ [Su et al., 2006]. The authors of the study [Su et al., 2006] suggested that the ionosphere plasma irregularities of another nature are responsible for the appearance of the mentioned mid-latitude peaks. Unfortunately, the search for the publications developing this idea was unsuccessful.

On the other hand, according to the results of Sidorova and Filippov [2012, 2014] the EPBs detected at much higher altitude, i.e. at the altitudes of the topside ionosphere $(\sim 972-1220 \mathrm{~km}$, ISS-b spacecraft), are more often recorded just in the mid-latitude region. These EPBs are detected at the topside ionosphere heights as the depletions in the $\mathrm{He}+$ density and can exist there for quite a long time [Sidorova and Filippov, 2014].

Thus, we have a number of the serious questions dealing with the features of the EPB latitudinal distributions with increasing the observation height. These issues need to be resolved. In this paper, we plan to study these issues in detail.

To advance this task, the detailed comparative analysis of the latitudinal histograms of the EPB occurrence probability obtained at the different altitudes will be performed. For this purpose, the data from ISS-b, ROCSAT-1 and AE-E spacecrafts with different orbital altitudes will be involved. It is worthy to mention that these satellite data are obtained in the years of high and maximal solar activity.

\section{Data: Equatorial plasma bubbles, EPBs}

The tabular summary $\sigma$ of the data used in this investigation is presented in the Table 1 .

\section{$2.1 \quad$ ISS-b data}

The histograms of the EPB latitudinal occurrence probability $\left(P_{E P B}\right)$ were obtained by using the $\mathrm{He}^{+}$ density depletion dataset [RRL, 1983, 1985]. The dataset was based on the ISS-b spacecraft operation during the high solar activity period $\left(1978-1979, F_{10.7} \sim 150-220\right)$. The ISS-b spacecraft was launched into a nearly circular orbit $(\sim 972-1220 \mathrm{~km})$ with an orbital period of $\sim 107 \mathrm{~min}$, and with an inclination angle of $\sim 70^{\circ}$ (Table 1$)$.

Note that the results of the $\mathrm{He}^{+}$density depletion observations were used for the $P_{E P B}$ calculation. According to Sidorova [2007, 2008] such depletions are interpreted as"fossil" signatures of the EPBs 
International Journal of Electronics

Vol:8, Issue:1, 2021 and Applied Research (IJEAR)

Table 1: The data used for the comparison

\begin{tabular}{|c|c|c|c|c|c|c|c|}
\hline Publication & $\begin{array}{l}\text { Space } \\
\text { craft }\end{array}$ & Parameter & $\begin{array}{c}\mathrm{LT} \\
\text { Interval }\end{array}$ & $\begin{array}{l}\text { Altitude } \\
(\mathrm{km})\end{array}$ & $\begin{array}{c}\text { Incilnation/ } \\
\text { latitudinal } \\
\text { Covering }\end{array}$ & Year & $F_{10.7}$ \\
\hline $\begin{array}{c}\text { Present } \\
\text { Investigation }\end{array}$ & ISS-b & $P_{E P B}$ & $\begin{array}{c}1800- \\
0600\end{array}$ & $\begin{array}{c}\sim 972- \\
1220\end{array}$ & $\begin{array}{c}-70^{0} / \pm 60^{0} \\
\text { DIPLAT }\end{array}$ & $\begin{array}{c}1978- \\
1979\end{array}$ & $\begin{array}{c}150- \\
220\end{array}$ \\
\hline Su et al. [2006] & ROCSAT-1 & $P_{\sigma<0.3 \%}$ & $\begin{array}{c}1800- \\
0600\end{array}$ & $\sim 600$ & $\begin{array}{c}35^{0} / \pm 55^{0} \\
\text { DIPLAT }\end{array}$ & $\begin{array}{c}1999- \\
2004\end{array}$ & $\begin{array}{c}107- \\
182\end{array}$ \\
\hline Kil and Heelis [1998] & $\mathrm{AE}-\mathrm{E}$ & $P_{\sigma<1 \%}$ & $\begin{array}{c}19: 00- \\
04: 00\end{array}$ & $\begin{array}{c}\sim 300- \\
\quad 475\end{array}$ & $\begin{array}{c}19.7^{0} / \pm 40^{0} \\
\text { DIPLAT }\end{array}$ & $\begin{array}{c}1978- \\
1980\end{array}$ & $\sim 185$ \\
\hline
\end{tabular}

Table 2: Season periods of the observations

\begin{tabular}{ccccc}
\hline Publication & Winter & Vernal & Summer & Autumn \\
\hline Present & November- & February- & May- & August- \\
Investigation & January & April & June & October \\
{$[$ Su et al., 2006] } & December & March & June & September \\
[Kil and Heelis, 1998] & November- & February - & May - & August - \\
& January & April & July & October \\
\hline
\end{tabular}

seen in the topside ionosphere in the $\mathrm{He}^{+}$density. (The idea is well supported by the numerical estimations [Sidorova and Filippov, 2014] and validated by the statistical studies [Sidorova, 2004, 2007, 2008; Sidorova and Filippov, 2012].) Bennett mass spectrometer (3 stage, 3-5 cycle Bennett tube; mass range of 1-20 AMU; grid separation of $0.3 \mathrm{~cm}$; RF frequency of $14 \mathrm{MHz}, 6 \mathrm{MHz}$ ) was used for the $\mathrm{He}^{+}$ density measurements. Instrumental resolution $(\mathrm{R} \sim 50)$ was sufficient to clearly identify the $\mathrm{He}^{+}$ions [Iwamoto, 1997]. For this study we have taken into account only the well-pronounced $\mathrm{He}^{+}$density depletions. The depletions from several times to two orders of magnitude of the background plasma density were under considerations only. As a rule, such depletions are characterized by the cross section within $2^{0}$ to $10^{0}$ latitude (or $\sim 200-1000 \mathrm{~km}$ ).

The $P_{E P B}$ histograms were calculated for each season (winter, vernal, summer, autumn). Two-year period (18 August, 1978 - 31 December, 1979) [RRL, 1983, 1985] was used for the calculations. Seasonal values of $\mathrm{EPB}$ were obtained as pattern averaged over the 3-month interval of the observations (Table 2). The only exception was the summer season for which the $P_{E P B}$ values were calculated for two months (May-June) only, since the July data were absent.

Only the EPBs, which appear during the prolonged magnetically quiet periods $\left(K_{p} \leqslant 3\right)$ at the latitudes of $\pm 60^{0}$ DIPLAT in the interval of 18-06 LT, were under consideration. The histograms of the EPB calculated for each dipole latitudinal bin of $5^{0}$ are shown in Figures 1a-4a. 


\subsection{ROCSAT-1 data}

For the comparison we have used the latitudinal variations of the EPB occurrence rates derived by $\mathrm{Su}$ et al. [2006] from the ion density $\left(\mathrm{N}_{i}\right)$ data obtained onboard the spacecraft ROCSAT-1. ROCSAT-1 spacecraft was launched into a nearly circular orbit $(\sim 600 \mathrm{~km})$ with an inclination angle of $\sim 35^{\circ}$ (Table $1)$. The EPBs detected from the fluctuations in $\left(\mathrm{N}_{i}\right)$ with the normalized index of $\sigma<0.3 \%$ were under consideration and used for the occurrence rate calculations $\left(P_{\sigma<0.3 \%}\right)$. (Let us recall that $\sigma=\frac{\Delta N_{i}}{\bar{N}_{i}}$ where i is the mean background ion density and $\bar{N}_{i}$ is the standard deviation of the background ion density.) The onboard instruments had a high spatial resolution, which allowed detection of the irregularities ranging in size from $7.5 \mathrm{~km}$ to $75 \mathrm{~km}$. The authors [Su et al., 2006] have used the EPB data collected during five years of the observations (1999-2004) covered the periods of the high and maximal solar activity $\left(F_{10.7} \sim 107-181\right)$ (Table 1$)$.

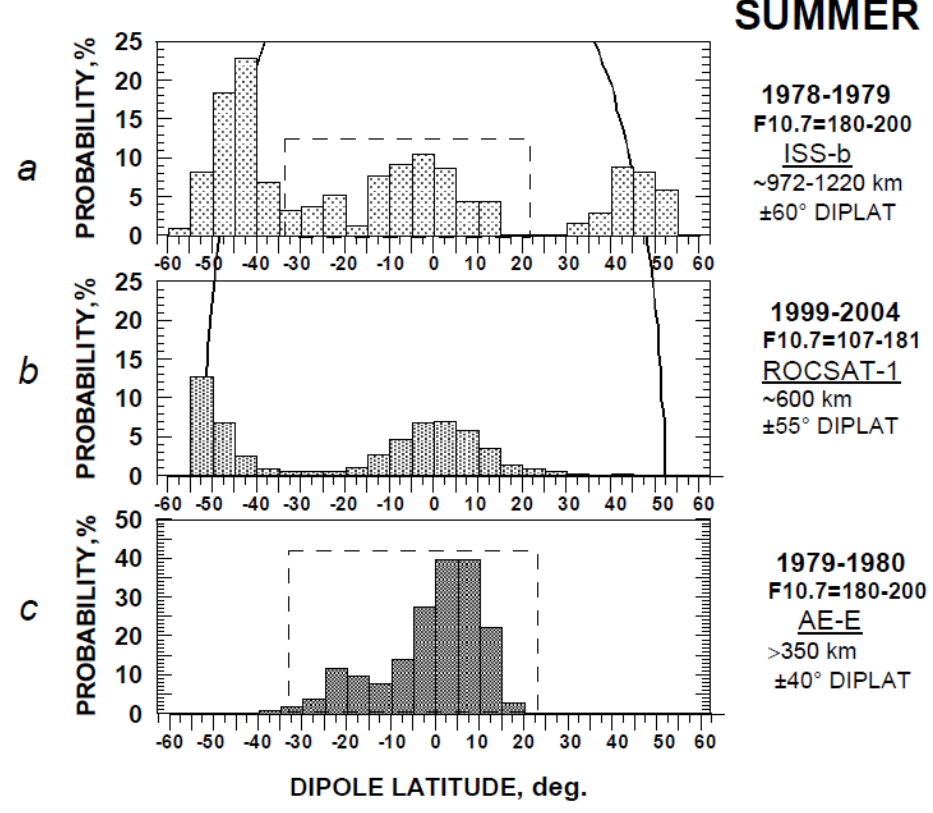

Figure 1: Histograms of the EPB occurrence probability versus dipole latitude for the summer season.(a)Histogram of $P_{E P B}$ obtained from ISS-b spacecraft data.The $P_{E P B}$ values are calculated for the $\pm 60^{0}$ DIPLAT latitude band, the latitude step is equal to $5^{0}$.(b) Histogram of $P_{\sigma<0.3 \%}$ obtained from the ROCSAT-1 spacecraft data [Su et al., 2006].(c) Histogram of $P_{\sigma<1 \%}$ obtained from the AE-E spacecraft data [Kil and Heelis, 1998]. The $P_{\sigma<1 \%}$ values are calculated for the $\pm 40^{0}$ DIPLAT latitude band, the latitude step is equal to $\pm 10^{0}$.

The histograms were presented as a pattern averaged over one typical month of each season (December, March, June and September) (Table 2). The EPB data were gathered in the period of 18-06 LT in 
the latitude range of $\pm 55^{0}$ DIPLAT. The $P_{\sigma<0.3 \%}$ histograms obtained in the $10^{0}$ dipole latitude bins are shown in Figures 1b-4b.

\section{$2.3 \quad$ AE-E data}

Also the histograms of the EPB latitudinal occurrence probability obtained by Kil and Heelis [1998] from the AE-E spacecraft $(\sim 300-475 \mathrm{~km})$ data were used for the comparison. The structures of EPB were detected from the $\mathrm{N}_{i}$ fluctuations. Only the $\mathrm{N}_{i}$ values with $\sigma<1 \%$ were taken for the occurrence rate calculations $P_{\sigma<1 \%}$. The instrumental characteristics and the data-selection techniques used in the study allowed identify the EPBs ranging in size from $100 \mathrm{~km}$ to $1000 \mathrm{~km}$.

We have used the $P_{\sigma<1 \%}$ histograms [Kil and Heelis, 1998] derived from the EPB data recorded at the altitudes $\sim 350-475 \mathrm{~km}$ only. The median values of $P_{\sigma<1 \%}$ were calculated over two-year observation period (1978-1980) characterized by the high level of solar activity $\left(F_{10.7} \sim 185\right)$.

The EPB data were gathered in the period of 19-04 LT in the latitude range of $\pm 40^{0}$ DIPLAT (Table 1). The values were calculated for each season as the median for three months of the observations (Table 2). The $P_{\sigma<1 \%}$ histograms plotted versus dipole latitude with a step of $10^{0}$ are shown in Figures 1c-4c.

\section{Comparative analysis}

The main issues we want to discuss in this paper are the variability of the EPB latitudinal distributions with altitude. For these aims let us compare the typical patterns of the mentioned distributions obtained at the different altitudes (Figures 1-4).

As was mentioned above, all EPB data used for the histogram calculations were obtained during high and maximal solar activity, i.e. under the similar heliophysical conditions. Note that these conditions are the most favorable for the EPB generation [Gentile et al., 2006].

If we consider the periods of solstices (summer, winter) (Figures 1,2), the histograms of the EPB latitudinal occurrence probability obtained at altitudes of $\sim 350-475 \mathrm{~km}$ (AE-E) are characterized by the dominance of the central (equatorial) maximum (Figure. 1c, 2c). There is a slight asymmetry in the shape of this maximum (the presence of the side "lobe"). It is clearly seen that the EPBs are more often generated in the winter hemisphere than in the summer one. However, this effect disappears at the altitudes of $\sim 600 \mathrm{~km}$ (ROCSAT-1) (Figures 1b-2b). On the other hand, other feature begins to appear: the additional maxima of the EP B occurrence probability appear in both hemispheres at the latitudes of $\pm\left(50^{0}-55^{0}\right)$ DIPLAT. These mid-latitude peaks show a significant increase in the EPB distributions obtained in the topside ionosphere, i.e. at the altitudes of $\sim 972-1220 \mathrm{~km}$ (ISS-b) (Figure 1a, 2a). Although, they no longer appear at the same latitudes but at the latitudes of $\pm\left(45^{0}-50^{\circ}\right)$ DIPLAT which are slightly shifted to the equator (Figures 1a, 2a). The reasons of this shift will be discussed in the next Section. Moreover, it is clearly seen that the amplitudes of these mid-latitude maxima are more significant in the winter hemisphere than in the summer one (Figures 1a, 1b, 2a, 2b). It is clearly seen also that at the altitudes of $\sim 972-1220 \mathrm{~km}$ (ISS-b) these peaks begin to dominate relative to the equatorial maximum (Figures 1a, 2a).

Note that we do not compare the histograms of the EPB latitudinal occurrence probability obtained at the different altitudes by absolute values. We believe that such comparison is not entirely correct, since the EPB data obtained from the different spacecrafts with the different measuring devices were under consideration. Moreover, the different approaches and methods of data processing were used to 


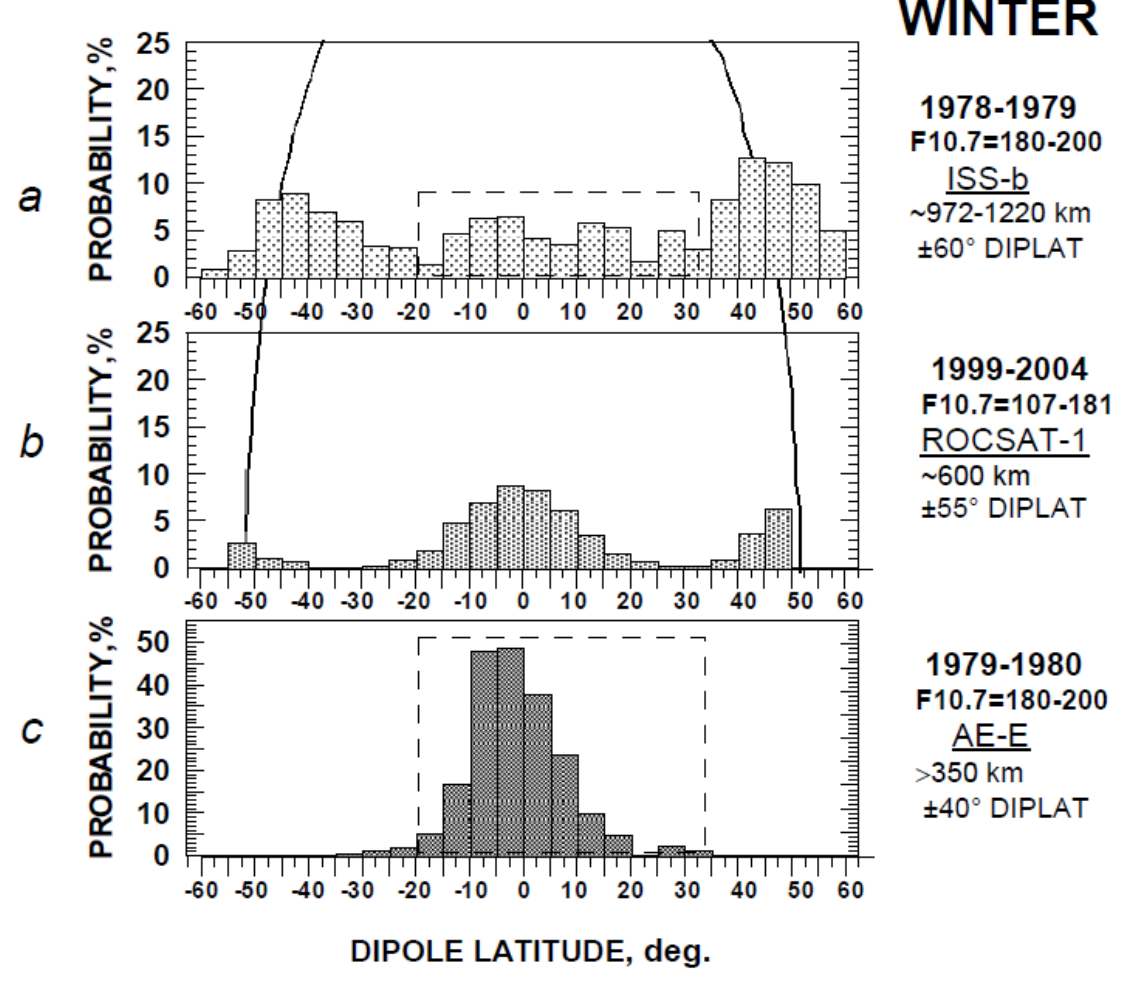

Figure 2: The same for the winter season only.

identify the EPB structures in the different studies.

If we consider the equinox periods (vernal, autumn) (Figures 3, 4), the histograms of the EPB latitudinal occurrence probability obtained at the altitudes of $\sim 350-475 \mathrm{~km}$ (AE-E) are also characterized by the dominance of the central (equatorial) maximum (Figure. 3c, 4c). Moreover, the additional midlatitude maxima appear in both hemispheres $\pm\left(50^{0}-55^{0}\right)$ DIPLAT, when the histograms from the higher altitudes (ROCSAT- $1, \pm 600 \mathrm{~km}$ ) are under consideration. These maxima show a significant increase relative to the equatorial maximum at the altitudes of $\pm 972-1220 \mathrm{~km}$ (ISS-b) (Figures 3a, 4a). And again, it should be pointed out that they no longer appear at the previous latitudes, but at the latitudes of $\pm\left(45^{0}-50^{0}\right)$ DIPLAT, which are slightly shifted to the equator (Figures 3a, 4a). There is a slight difference in the values of these maxima in different hemispheres, but this difference is not so pronounced compare to the difference in the solstice periods.

\section{Discussion}

As it was shown above, the significant changes in the shape of the EPB latitudinal occurrence rate accumulate with increasing the observation height (Figures 1-4). So, if the occurrence rates obtained at 


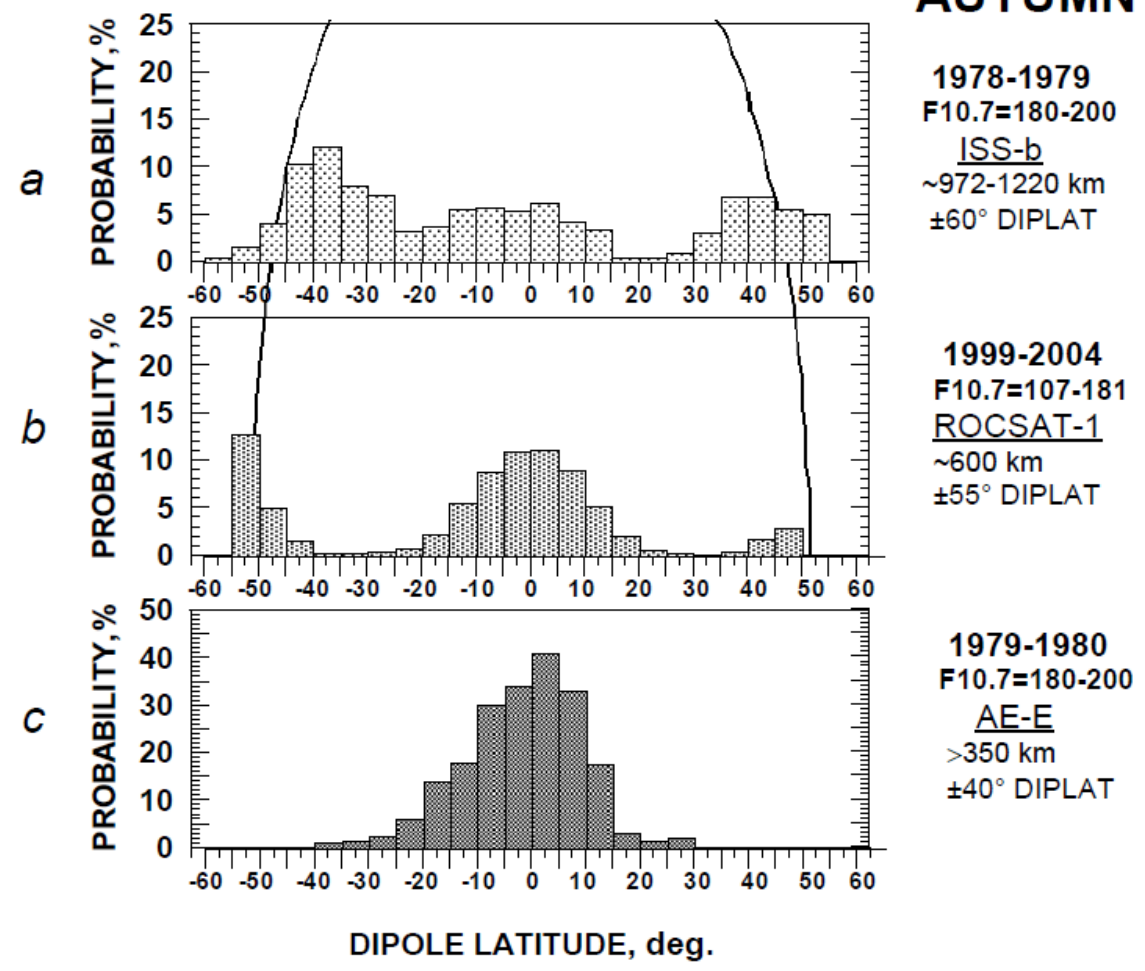

Figure 3: The same for the autumn season only.

the different altitudes show the basic similarity in the low and equatorial latitude region, they have the cardinal differences in the mid-latitude regions. Let's consider these trends and discuss the reasons of their development.

Initially, let's analyze the latitudinal histograms of the EPB occurrence probability obtained from the AE-E data at the altitudes of $\pm 350-475 \mathrm{~km}$ (Figures 1c-4c). Note that the lower boundary of this altitudinal interval is the heights of the bottom side of the F-region, where the EPB generation takes place. Moreover, it is well known also that the EPB generation occurs in the region of the equatorial latitudes. It reflects in the formation of the central maximum over the equatorial latitudes. However, it is clearly seen that the width of this maximum can reach quite significant sizes (up to $\pm\left(25^{0}-30^{0}\right)$ DIPLAT) in some seasons (see, e.g., Figures 1c, 3c). That means that the developing EPBs can be detected not only over the equator, but over the low-latitude region also. To explain the above, we should consider the main facts dealing with the processes of the EPB generation and evolution.

It is known that the EPBs are formed after sunset under the influence of the R-T plasma instability, which develops at the altitudes of the bottom side of the F-region $( \pm 300-400 \mathrm{~km})$. Another important condition for the EPB generation is a presence of the plasma seed perturbations. After sunset, i.e. after the evening pre-reversal enhancement of the $\mathrm{E} \times \mathrm{B}$ drift, the available seed perturbations begin to evolve and transform into the equatorial plasma bubbles (EPBs). It is evident that the just formed plasma 


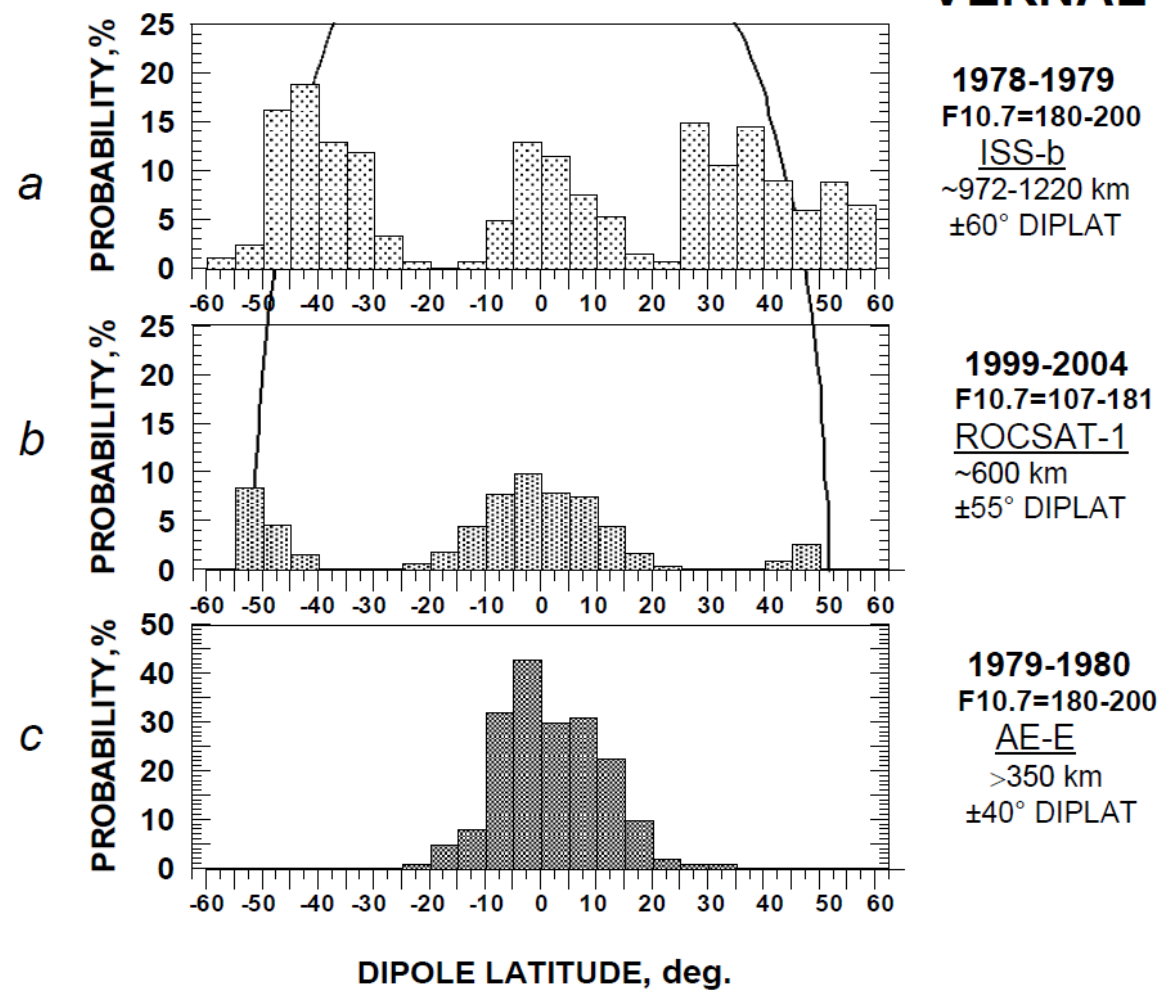

Figure 4: The same for the vernal season only.

bubbles are very small, since they are at the initial stage of their evolutionary development and uplifting. The vertical growth of the EPB is centered over the equator, which is responsible for equatorial maximum of the EPB occurrence rate (Figures $1 \mathrm{c}-4 \mathrm{c}$ ).

When the bubbles uplift higher, the bubbles extend due to the ambipolar diffusion along the magnetic tubes. (The illustration of the EPB development and uplifting is presented in Figure. 5.) Their extension becomes more and more significant in the process of their uplifting. So extended bubbles look like "bananas" with the extremities reaching the ionosphere heights possibly in both hemispheres. Such bubble structures could be detected by satellites as depletions not only above the equator but also in the low-latitudinal region $( \pm 350-475 \mathrm{~km}$, Figures $1 \mathrm{c}-4 \mathrm{c})$.

Another trend that begins to develop at the altitudes of $\pm 600 \mathrm{~km}$ (ROCSAT- 1 ) is the appearance of the additional maxima at the mid-latitudes of the both hemispheres (Figures 1b-4b). These maxima appear here at the latitudes of $\pm\left(50^{0}-55^{0}\right)$ DIPLAT. However, at the altitudes of $( \pm 972-1220 \mathrm{~km}$, ISS-b), they become more significant and slightly shift to the equator $\pm\left(45^{0}-50^{\circ}\right)$ DIPLAT. A reasonable question arises: why do these mid-latitude maxima in addition to the apparent equatorial maximum occur? 
Obviously, when the bubbles rise to the topside ionosphere heights, they can also be detected at the mid-latitudes. Really, it is clearly seen (Figure. 5) that when the apex part of the rising bubble is detected above the equator, their extremities spread along the magnetic tubes are detected in the lowand mid-latitudinal regions. (We should point that it is possible to detect both the bubble itself (its fragment) and the "noise cloud" of the small-scale plasma irregularities surrounding/accompanying this bubble [Abdu et al., 1983, 2000; Aggson et al., 1992; Tsunoda, 1980]. At first glance, this process should be reflected in the latitudinal distribution of the EPB as monotonically decaying one. However, this is not the case in reality.

It is known that the EPBs rising to the topside ionosphere altitudes begin to reach their maximal ("ceiling") altitudes. We should remind that the ceiling altitude is the altitude where the bubble stops rising, continues to exist for a while as a "fossil" bubble and then gradually disappears. According to the theory [Ossakow and Chaturvedi, 1978; Ott, 1978], the ceiling altitude of the plasma bubble is determined by the condition of the equality of the plasma density inside and outside the bubble. According to Burke et al. [1979] such bubbles are well detected even at the altitudes of $\sim 3500 \mathrm{~km}$. For example, if the bubble apex reaches after several hours of rise its ceiling altitude, e.g., $\sim 3000 \mathrm{~km}$ (Figure.5), then the halves of the bubble, descending along the magnetic field tube, reach the altitudes of the satellite observations, e.g., $\sim 1000 \mathrm{~km}$ (ISS-b) at the latitude of $\sim \pm 30^{\circ}$ DIPLAT and $\sim 600 \mathrm{~km}$ (ROCSAT- 1 ) at the latitude of $\sim \pm 33^{0}$ DIPLAT. This bubble lost ability to rise decelerates, remains on the specified magnetic field tube for several hours and then gradually collapses. As a result, the bubbles that stopped at their ceiling altitudes accumulate, leading to the gradual growth of the additional mid-latitude maxima in the latitudinal distribution of the EPB occurrence probability $(\sim 600 \mathrm{~km}$, Figures 1b-4b; $972-1220 \mathrm{~km}$, Figures 1a-4a). Moreover, the small latitudinal shift of these maxima to the equator with increasing the observation height is associated exclusively with the spatial course of the magnetic field tube. (The spatial course of the magnetic field tubes are shown by the arc-shaped curves in Figures $1 \mathrm{a}, \mathrm{b}-4 \mathrm{a}, \mathrm{b}$.) And the fact that these maxima become more pronounced at the altitudes of $\sim 972-1220 \mathrm{~km}$ means the accumulation of the increasing number of the EPBs reaching their ceiling altitudes (Figures 1a-4a).

As it was pointed out above, the values of the mid-latitude maxima in the histograms obtained at the altitudes of $\sim 972-1220 \mathrm{~km}$ (ISS-b) are comparable or begin to dominate with respect to the equatorial maximum (Figures 1a-4a). It results from the latitudinal variations of the background $\mathrm{He}^{+}$density in the topside ionosphere. Latitudinal $\mathrm{He}^{+}$density profile has deep equatorial trough (the density decrease is 5 times and more) and crests of this trough at the latitudes of $\pm\left(20^{\circ}-400\right)$ DIPLAT [Chandra, 1975; Chandra et al., 1970; Taylor Jr et al., 1970]. (We should remind that the EPB data obtained from the ISS-b measurements were detected as the depletions in the He+ density.) Accordingly, the EPB detection in the region of the equatorial $\mathrm{He}^{+}$density trough is difficult due to the small difference between the background $\mathrm{He}+$ density and the $\mathrm{He}+$ density inside the bubble. (For example, the equatorial maximum is particularly poorly detected in winter (Figure 2a).) On the other hand, the EPBs are the most easily detected in the region of the crests in the $\mathrm{He}^{+}$density, since this difference is significant.

Let's discuss the features of the latitudinal distributions of the EPB occurrence rate in the different seasons.

If the periods of the solstice (summer, winter) (Figures 1-2) are under consideration, it is easy to notice that the shape of the equatorial maximum has more or less pronounced asymmetry at the altitudes of the bottom side of the F-region $(\sim 350-475 \mathrm{~km})$. So, the central peak of the occurrence rate has a side "lobe" in the summer season in the Southern (winter) hemisphere (Figure 1c). 


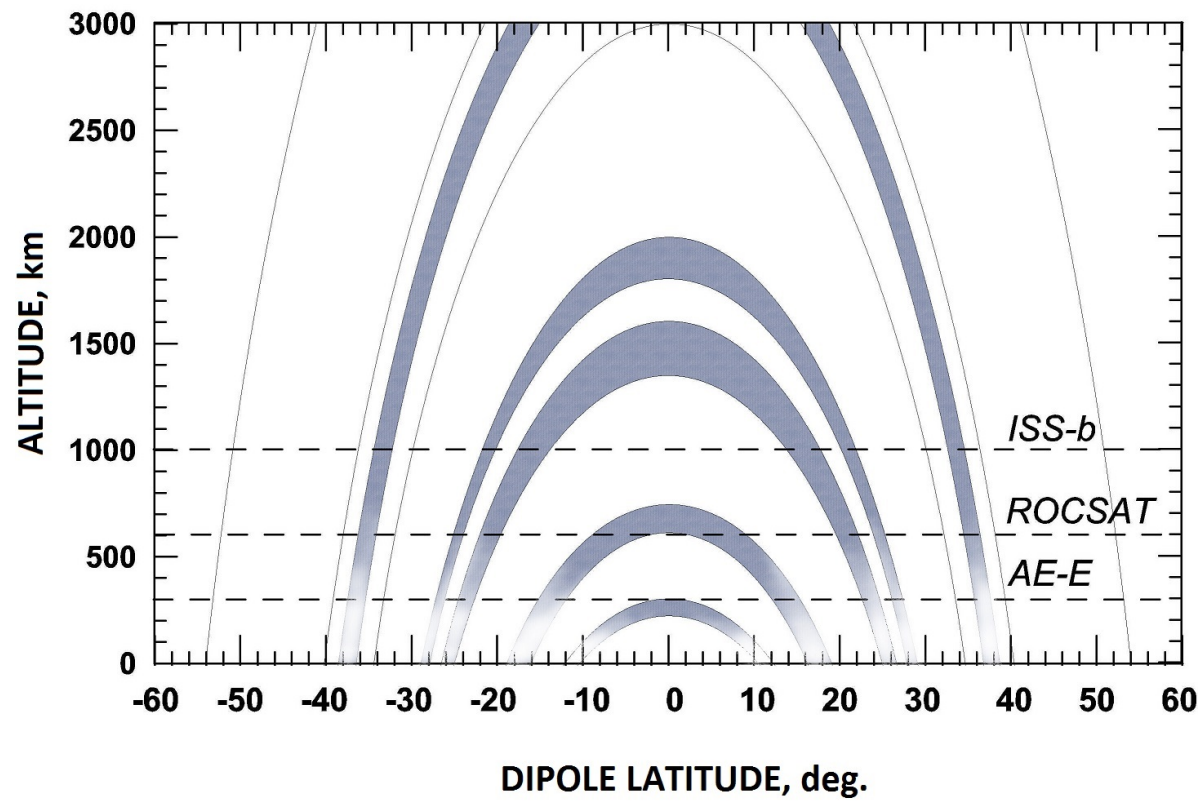

Figure 5: Schematic representation of the EPB evolution with respect to the magnetic field lines, dipole latitude and altitude. The horizontal lines show the approximate altitudes of the ISS-b $(\sim 972-1220 \mathrm{~km})$, ROCSAT-1 $(\sim 600 \mathrm{~km})$ and AE-E $(\sim 350-475 \mathrm{~km})$ spacecrafts

Moreover, the same is developing in the winter season in the Northern hemisphere (Figure 2c). It means that there are more favorable conditions for the EPB development in the winter hemisphere than in summer one. Moreover, the additional maxima appearing in the middle latitudes of the topside ionosphere $(\sim 600 \mathrm{~km}$ and $\sim 972-1220 \mathrm{~km})$ show the maximal growth again in the winter hemisphere (Figures 1a, b - 2a, b). These results are in the good accordance with the well known seasonal features found in the EPB observation (see, e.g., [Gentile et al., 2006]). Thus, according to the maps of the seasonal-longitudinal EPB occurrence probability obtained during the years of the increased solar activity (1989-1992, DMSP spacecrafts, $\sim 848 \mathrm{~km}$ ) ( [Gentile et al., 2006]) and the Figure 4 therein) the plasma bubbles most often appear in the winter, vernal and autumn seasons. The EPB development is least frequent in the summer months. This can be easily illustrated by the Figure. 5. So, the most favorable conditions for the development of the rising plasma bubbles (their fragments) take place in the winter hemispheres. And, conversely, the plasma bubble development is strongly suppressed in the summer hemisphere.

It was noticed previously that there are the similar parts (highlighted by the rectangles) in the latitudinal patterns of the EPB occurrence probability at the altitudes of $\sim 350-475 \mathrm{~km}$ and $\sim 972-1220 \mathrm{~km}$. The similarity is seen in the repetition of the side "lobe" of the equatorial maximum (Figures 1a and 1c; Figures 2a and 2c). It is most pronounced in summer in the Southern hemisphere and less pronounced (due to the poorly developed equatorial maximum) in winter in the Northern hemisphere. In the meantime, the mentioned side "lobe" disappears in the latitudinal histograms obtained at the $\sim 600 \mathrm{~km}$. It is reasonable to ask about the possible causes of this phenomenon.

The fact is that the EPB structures are detected from the ISS-b data as the plasma regions with depleted $\mathrm{He}^{+}$density, i. e. the EPBs are detected from the fluctuation in the single ion component. 
According to the AE-A and ROCSAT-1 spacecraft data the EPBs are detected from the fluctuation in the total ion density $\left(\mathrm{N}_{i}\right)$. It is well known that the oxygen ions $\left(\mathrm{O}^{+}\right)$dominate in the total ion density and the background $\mathrm{O}^{+}$density steadily decreases with increasing the $\mathrm{F} 2$ region altitude (see, e. g., [Bryunelli and Namgaladze, 1988]). It makes increasingly difficult to identify the EPB structure in the $\mathrm{N}_{i}\left(\mathrm{O}^{+}\right)$density, since the depleted $\mathrm{N}_{i}\left(\mathrm{O}^{+}\right)$density inside the plasma bubble becomes gradually equalized with the background $\mathrm{O}^{+}$density outside the plasma bubble. Growing problems in the EPB registration lead to the disappearance of the details in the latitudinal histograms of the EPB occurrence rate (Figures $1 \mathrm{~b}-4 \mathrm{~b})$. At last, the EPBs reaching the upper topside ionosphere altitudes $(\sim 972-1220 \mathrm{~km})$ become almost "invisible" if we try to detect them in the total ion $\left(\mathrm{N}_{i}\right)$ or electron density $(\mathrm{Ne})$ because $\mathrm{N}_{e} \cong \mathrm{N}_{i}$. So, according to the ISS-b spacecraft data the EPBs are detected as the Ne density depletions in $3 \%$ of the passes only (46 cases in 1700 passes) [Maruyama and Matuura, 1980]. However, according to the same spacecraft data they are still "visible" but they are "visible" in the minor ion component $\left(\mathrm{He}^{+}\right)$[Sidorova, 2004, 2007, 2008]. (We should remind that the $\mathrm{He}^{+}$density in the EPB plasma content is determined by the background $\mathrm{He}^{+}$density at the altitudes of the EPB generation, where the $\mathrm{He}^{+}$ions are present in very small amounts. For example, according to the Arecibo radar measurements [Wilford et al., 2003], the absolute $\mathrm{He}^{+}$density is $2.5 \times 10^{2}-3 \times 10^{3} \mathrm{~cm}^{-3}$ at the altitudes of $350-450 \mathrm{~km}$ (high solar activity, October 2001).) In other words, the plasma bubble becomes "visible" at the upper topside ionosphere altitudes $(\sim 972-1220 \mathrm{~km})$ as the $\mathrm{He}^{+}$density depletion. It becomes "visible" since the surrounding background $\mathrm{He}^{+}$density strongly increases at the upper topside altitudes and may even be dominant [Heelis et al., 1990]. Such background $\mathrm{He}^{+}$density shows contrast with insignificant small $\mathrm{He}^{+}$density content inside the plasma bubble [Sidorova, 2004, 2007, 2008; Sidorova and Filippov, 2012]. According to the numerical calculations, the EPBs detected as the He+ density depletions are fairly stable structures [Sidorova and Filippov, 2014].

For this reason, the latitudinal patterns of the EPB occurrence probability recorded at the altitudes of $\sim 350-475 \mathrm{~km}$ near the EPB generation altitudes are fairly accurately reflected at the altitudes of the upper topside ionosphere $(\sim 972-1220 \mathrm{~km})$ and appears "blurred" ones at the intermediate altitudes $(\sim 600 \mathrm{~km})$.

If the periods of the equinox (vernal, autumn) (Figures 3-4) are under consideration, it is clearly seen that the latitudinal EPB occurrence rate at the altitudes of the bottom side of the F-region $(\sim 350-475 \mathrm{~km})$ is also characterized by the dominance of the equatorial maximum (Figures 3c-4c). As it was mentioned earlier, the mid-latitude maxima appear at the altitudes of $\sim 600 \mathrm{~km}$ in addition to the equatorial maximum (Figures $3 \mathrm{~b}-4 \mathrm{~b}$ ). Their values are comparable with the value of the equatorial maximum here. However, their values begin to dominate at the altitudes of $\sim 972-1220 \mathrm{~km}$ (Figures 3a-4a). There is also a slight difference in the values of the mid-latitude maxima recorded in the different hemispheres (Figures 3a-4a). However, this difference is not so significant in comparison with such difference observed during the solstices. This result is quite expected since the both hemispheres have approximately the same conditions for the EPB development in the equinox seasons [Gentile et al., 2006].

So, it is possible to conclude that the revealed variability of the EPB latitudinal distributions with altitude has its logical explanation within the framework of the common mechanism of the EPB generation and evolution. In addition, the obtained seasonal features of the EPB latitudinal distributions are in good agreement with previously known seasonal characteristics of the EPB observations 


\section{Conclusion}

The characteristics of the variability of the histograms of the EPB latitudinal occurrence probability with altitude was studied. For this purpose, a detailed comparative analysis of the latitudinal histograms of the EPB occurrence probability obtained at the different altitudes was performed. The data from the ISS-b $(\sim 972-1220 \mathrm{~km})$, ROCSAT-1 $(\sim 600 \mathrm{~km})$ and AE-E $(\sim 350-475 \mathrm{~km})$ spacecrafts were used. The EPB structures were identified from the ISS-b data as the $\mathrm{He}^{+}$density depletions, and from the ROCSAT-1 and AE-E data as the ion density $\left(\mathrm{N}_{i}\right)$ depletions. Different seasons during the years of the high and maximal solar activity were under consideration. The main findings from this study are the following:

1. As the observation height increases from $\sim 600 \mathrm{~km}$ to $\sim 1000 \mathrm{~km}$, the mid-latitude maxima in addition to the equatorial maximum develop in the histograms of the EPB latitudinal occurrence probability. This feature is most pronounced in both the hemispheres of the equinox periods and in the winter hemisphere.

2. At the altitudes of the upper topside ionosphere $(\sim 972-1220 \mathrm{~km}$, ISS-b), the values of the midlatitude maxima of the EPB occurrence probability become comparable or begin to dominate in relation to the equatorial maximum. This feature is typical for all seasons of the observations.

3. It was found that the latitudinal positions of the mid-latitude maxima of the EPB occurrence probability shift to the equator with increasing the observation heights. The latter is in good accordance with the variability of the latitudinal courses of the magnetic field tubes along which the plasma bubbles that have already reached their ceiling heights are stretched.

\section{Acknowledgement}

I express my gratitude to ISS Research and Operation Committee (Japan) for providing the ISS-b satellite data.

\section{References}

Abdu, M., De Medeiros, R., Sobral, J., and Bittencourt, J. (1983). Spread F plasma bubble vertical rise velocities determined from spaced ionosonde observations. Journal of Geophysical Research: Space Physics, 88(A11):9197-9204.

Abdu, M., Sobral, J., and Batista, I. (2000). Equatorial spread F statistics in the American longitudes: Some problems relevant to ESF description in the IRI scheme. Advances in Space Research, 25(1):113124.

Aggson, T., Maynard, N., Hanson, W., and Saba, J. L. (1992). Electric field observations of equatorial bubbles. Journal of Geophysical Research: Space Physics, 97(A3):2997-3009.

Bryunelli, B. and Namgaladze, A. (1988). Ionosphere Physics (in Russian), page 557. Nauka, Moscow, Russia.

Burke, W., Donatelli, D., Sagalyn, R., and Kelley, M. (1979). Low density regions observed at high altitudes and their connection with equatorial spread F. Planetary and space Science, 27(5):593-601.

Chandra, S. (1975). The equatorial helium ion trough and the geomagnetic anomaly. Journal of Atmospheric and Terrestrial Physics, 37(2):359-367. 
Chandra, S., Troy Jr, B., Donley, J., and Bourdeau, R. (1970). Ogo 4 observations of ion composition and temperatures in the topside ionosphere. Journal of Geophysical Research, 75(19):3867-3878.

Gentile, L., Burke, W., and Rich, F. (2006). A climatology of equatorial plasma bubbles from DMSP 1989-2004. Radio Science, 41(5).

Heelis, R., Hanson, W., and Bailey, G. (1990). Distributions of $\mathrm{He}^{+}$at middle and equatorial latitudes during solar maximum. Journal of Geophysical Research: Space Physics, 95(A7):10313-10320.

Iwamoto, I. (1997). A study on the ion composition of the topside ionosphere by satellite-borne mass spectrometers. Communications Research Laboratory, Journal, 44(1):11-186.

Kil, H. and Heelis, R. (1998). Global distribution of density irregularities in the equatorial ionosphere. Journal of Geophysical Research: Space Physics, 103(A1):407-417.

Maruyama, T. and Matuura, N. (1980). Global distribution of occurrence probability of echoes based on ISS-b observation. Radio Research Laboratory, Journal, 27:201-216.

Ossakow, S. L. and Chaturvedi, P. (1978). Morphological studies of rising equatorial spread F bubbles. Journal of Geophysical Research: Space Physics, 83(A5):2085-2090.

Ott, E. (1978). Theory of Rayleigh-Taylor bubbles in the equatorial ionosphere. Journal of Geophysical Research: Space Physics, 83(A5):2066-2070.

RRL (1983). Summary plots of ionospheric parameters obtained from ionosphere sounding satellite-b. Technical report, Tokyo: Radio Research Laboratories Ministry of Posts and Telecommunications. Vol. $1-3$.

RRL (1985). Summary plots of ionospheric parameters obtained from ionosphere sounding satellite-b. Technical report, Tokyo: Radio Research Laboratories Ministry of Posts and Telecommunications. Vol.4.

Sidorova, L. (2004). $\mathrm{He}^{+}$density topside modeling based on ISS-b satellite data. Advances in Space Research, 33(6):850-854.

Sidorova, L. (2007). Plasma bubble phenomenon in the topside ionosphere. Advances in Space Research, $39(8): 1284-1291$.

Sidorova, L. (2008). Topside ionosphere bubbles, seen as $\mathrm{He}^{+}$density depletions: connection with ESF, vertical plasma drift, thermosphere wind and solar activity. 37th COSPAR Scientific Assembly, 37:2894.

Sidorova, L. and Filippov, S. (2012). Topside ionosphere $\mathrm{He}^{+}$density depletions: Seasonal/longitudinal occurrence probability. Journal of Atmospheric and Solar-Terrestrial Physics, 86:83-91.

Sidorova, L. and Filippov, S. (2014). Plasma bubbles in the topside ionosphere: Estimations of the survival possibility. Journal of Atmospheric and Solar-Terrestrial Physics, 119:35-41.

Su, S.-Y., Liu, C., Ho, H., and Chao, C. (2006). Distribution characteristics of topside ionospheric density irregularities: Equatorial versus midlatitude regions. Journal of Geophysical Research: Space Physics, $111(\mathrm{~A} 6)$.

Taylor Jr, H., Mayr, H., and Brinton, H. (1970). Observations of hydrogen and helium ions during a period of rising solar activity. Space Research $X$, pages $663-678$. 
Tsunoda, R. T. (1980). Magnetic-field-aligned characteristics of plasma bubbles in the nighttime equatorial ionosphere. Journal of Atmospheric and Terrestrial Physics, 42(8):743-752.

Wilford, C., Moffett, R., Rees, J., Bailey, G., and Gonzalez, S. (2003). Comparison of the He ${ }^{+}$layer observed over arecibo during solar maximum and solar minimum with ctip model results. Journal of Geophysical Research: Space Physics, 108(A12).

Woodman, R. F. and La Hoz, C. (1976). Radar observations of f region equatorial irregularities. Journal of Geophysical Research, 81(31):5447-5466. 